\title{
Hepatic magnetic resonance T1-mapping and extracellular volume fraction compared to shear-wave elastography in pediatric Fontan-associated liver disease
}

\author{
Charlotte de Lange ${ }^{1,2}$ (D) $\cdot$ Karl Julius Thrane ${ }^{1} \cdot$ Kristian S. Thomassen $^{1} \cdot$ Oliver Geier $^{3} \cdot$ Bac Nguyen $^{1} \cdot$ \\ Anders Tomterstad ${ }^{1} \cdot$ Lil-Sofie Ording Müller ${ }^{1} \cdot$ Erik Thaulow $^{4,5} \cdot$ Runar Almaas $^{6} \cdot$ Gaute Døhlen $^{4}$. \\ Kathrine Rydén Suther ${ }^{1}$ - Thomas Möller ${ }^{4}$
}

Received: 11 February 2020 / Revised: 10 June 2020 / Accepted: 10 August 2020 / Published online: 9 October 2020

(C) The Author(s) 2020

\begin{abstract}
Background Children with Fontan circulation are at risk of developing hepatic fibrosis/cirrhosis. Reliable noninvasive monitoring techniques are lacking or under development.

Objective To investigate surrogate indicators of hepatic fibrosis in adolescents with Fontan circulation by evaluating hepatic magnetic resonance (MR) T1 mapping and extracellular volume fraction measurements compared to US shear-wave elastography.

Materials and methods We analyzed hepatic native T1 times and extracellular volume fractions with modified Look-Locker inversion recovery. Liver stiffness was analyzed with shear-wave elastography. We compared results between 45 pediatric patients ages $16.7 \pm 0.6$ years with Fontan circulation and 15 healthy controls ages $19.2 \pm 1.2$ years. Measurements were correlated to clinical and hemodynamic data from cardiac catheterization.

Results MR mapping was successful in 35/45 patients, revealing higher hepatic T1 times (774 \pm 44 ms) than in controls (632 $\pm 52 \mathrm{~ms} ; P<0.001)$ and higher extracellular volume fractions $(47.4 \pm 5.0 \%)$ than in controls $(34.6 \pm 3.8 \% ; P<0.001)$. Liver stiffness was $1.91 \pm 0.13 \mathrm{~m} / \mathrm{s}$ in patients vs. $1.20 \pm 0.10 \mathrm{~m} / \mathrm{s}$ in controls $(P<0.001)$. Native T1 times correlated with central venous pressures $(\mathrm{r}=0.5, P=0.007)$. Native $\mathrm{T} 1$ was not correlated with elastography in patients $(\mathrm{r}=0.2, P=0.1)$ or controls $(\mathrm{r}=-0.3, P=0.3)$. Extracellular volume fraction was correlated with elastography in patients $(\mathrm{r}=0.5, P=0.005)$ but not in controls $(\mathrm{r}=0.2, P=0.6)$. Conclusion Increased hepatic MR relaxometry and shear-wave elastography values in adolescents with Fontan circulation suggested the presence of hepatic fibrosis or congestion. Central venous pressure was related to T1 times. Changes were detected differently with MR relaxometry and elastography; thus, these techniques should not be used interchangeably in monitoring hepatic fibrosis.
\end{abstract}

Keywords Adolescents $\cdot$ Chronic liver disease $\cdot$ Fontan procedure $\cdot$ Liver $\cdot$ Magnetic resonance imaging $\cdot$ Ultrasonographic elastography

Electronic supplementary material The online version of this article (https://doi.org/10.1007/s00247-020-04805-y) contains supplementary material, which is available to authorized users.

Charlotte de Lange

charlotte.de.lange@vgregion.se; charlotte.delange@medisin.uio.no

1 Division of Radiology and Nuclear Medicine, Section of Paediatric Radiology, Oslo University Hospital, Oslo, Norway

2 Department of Radiology and Clinical Physiology, Queen Silvia Children's Hospital, Sahlgrenska University Hospital, Rondv 10, S-41615 Göteborg, Sweden
Department of Physics, Oslo University Hospital, Oslo, Norway

4 Department of Paediatric Cardiology, Oslo University Hospital, Oslo, Norway

5 Institute of Clinical Medicine, University of Oslo, Oslo, Norway

6 Department of Paediatric Research and Division of Paediatric and Adolescent Medicine, Oslo University Hospital, Oslo, Norway 


\section{Introduction}

The Fontan operation was introduced more than 40 years ago as a lifesaving treatment for univentricular-type heart defects. This procedure creates an artificial circulation with two serial capillary beds by connecting the caval veins to the pulmonary artery [1]. In the Fontan circulation, transpulmonary blood flow is driven by chronically elevated central venous pressure, which leads to end-organ complications on both visceral organs and lymphatic drainage [2, 3]. In adults, Fontanassociated liver disease, characterized by fibrosis/cirrhosis and increased cancer risk, is a major concern. In pediatric patients, the prevalence and impact of liver disease are less well explored [3-5].

Noninvasive methods for monitoring liver fibrosis are lacking or under development. Liver biopsy is considered the gold standard method for assessing other structural liver diseases. However, the heterogeneous distribution of fibrosis in Fontan liver disease often results in non-representative biopsy samples [4, 6-9]; hence, estimating the risk of complication, this invasive procedure is only justified in select cases. Current international recommendations for evaluating these patients are mainly based on expert consensus [3,5]. Thus, evidence for suitable imaging techniques is urgently needed [3].

A promising new technique, MR T1 mapping with calculation of extracellular volume fraction, is routinely used in the detection of myocardial fibrosis [10-12]. Recently, this technique has been employed in the liver to diagnose adult noncongestive chronic liver disease to grade fibrosis, instead of histological grading [13-15]. Alternatively, shear-wave elastography performed with ultrasound (US) or MR can measure liver stiffness as a surrogate for fibrosis. US shear-wave elastography is simpler and less resource-demanding than $\mathrm{MR}$, and is routinely used to assess adult chronic liver disease $[16,17]$. Few studies have employed hepatic MR T1 mapping or elastography to evaluate fibrosis or congestion in people with Fontan circulation [18-22]. We hypothesized that adolescents with Fontan circulation might display elevations in fibrosis markers, based on MR T1 mapping, extracellular volume fraction, and US shear-wave elastography. We compared MR relaxometry to elastography in adolescents with a Fontan circulation and investigated the relationship between these measurements and clinical and hemodynamic parameters.

\section{Materials and methods}

This prospective study was approved by the regional institutional ethics board and was compliant with Health Insurance Portability and Accountability Act. It was registered at ClinicalTrials.gov (NCT02378857). Written informed consent was obtained from all patients, control individuals, or their caretakers.

\section{Study participants}

Adolescents with Fontan circulation, ages 15-17 years, were recruited from a follow-up program conducted at Oslo University Hospital that consisted of a comprehensive diagnostic workup prior to transition to adult care. These patients were consecutively included from March 2015 through December 2018. During a 4-day hospitalization, patients underwent a structured diagnostic workup, including MR of the liver/spleen and US elastography, performed on two consecutive days. Hepatic serological markers were sampled on Day 2, and cardiac catheterization was performed under general anesthesia/deep sedation on Day 3. Demographic, clinical and catheterization data were recorded. Catheterization data included central venous pressure measured in the conduit/ inferior caval vein, liver vein wedge pressure, and peak systolic and end-diastolic ventricular pressures.

For the control group, we recruited healthy individuals with a hospital-wide and internet-based announcement, by communications among hospital employee families and via social networks. For ethical reasons, the controls had to be $\geq 18$ years to consent to contrast agent exposure; thus, the age range was different between study groups.

\section{Magnetic resonance imaging}

Children with contraindications for MR or non-diagnostic T1 mapping were excluded from this examination and further analyses. The presence of ascites was recorded. After a 3-h fast, patients and controls underwent identical scanning protocols with a 1.5 -tesla $(\mathrm{T})$ system (Magnetom Aera; Siemens Healthcare, Erlangen, Germany). A coronal T2 half-Fourier single-shot turbo spin-echo and axial 3-D T1-W volumetric interpolated breath-hold examination were performed over the liver. An axial modified Look-Locker inversion recovery, with inline motion correction, was performed in the liver mid-section. Both exams were performed before and $15 \mathrm{~min}$ after intravenously administering $0.2 \mathrm{mmol} / \mathrm{kg}$ gadoterate meglumine (Dotarem; Guerbet, Villepinte, France). Before contrast injection, an axial $\mathrm{T} 2 *$ gradient multi-echo sequence was performed. Scan parameters and MR protocol details are shown in Online Supplementary Material 1.

\section{Magnetic resonance image post-processing}

$\mathrm{T} 1$ and $\mathrm{T} 2 *$ relaxation times were measured on parametric maps derived from source images on a Siemens Syngo satellite workstation. Five circular regions of interest $\left(2.0-2.5 \mathrm{~cm}^{2}\right)$ were placed in the periphery of both liver lobes and one was placed in the spleen $\left(2.5-3.0 \mathrm{~cm}^{2}\right)$ (Figs. 1 and 2). We took care to avoid major vessels and the hepatic surface; motion artifacts were corrected. Post-contrast T1 times were derived 
Fig. 1 Images of shear-wave elastography and native MR T1 mapping in an 18-year-old male control subject. a Shear-wave elastography. The blue color in the region of interest (yellow circle) indicates a normal value $<1.4 \mathrm{~m} / \mathrm{s}$. b Native MR T1 map shows a relatively uniform color; white circles indicate five regions of interest in the liver (1-5), one in the spleen (6), one in the inferior caval vein (7) and one in the aorta $(8)$
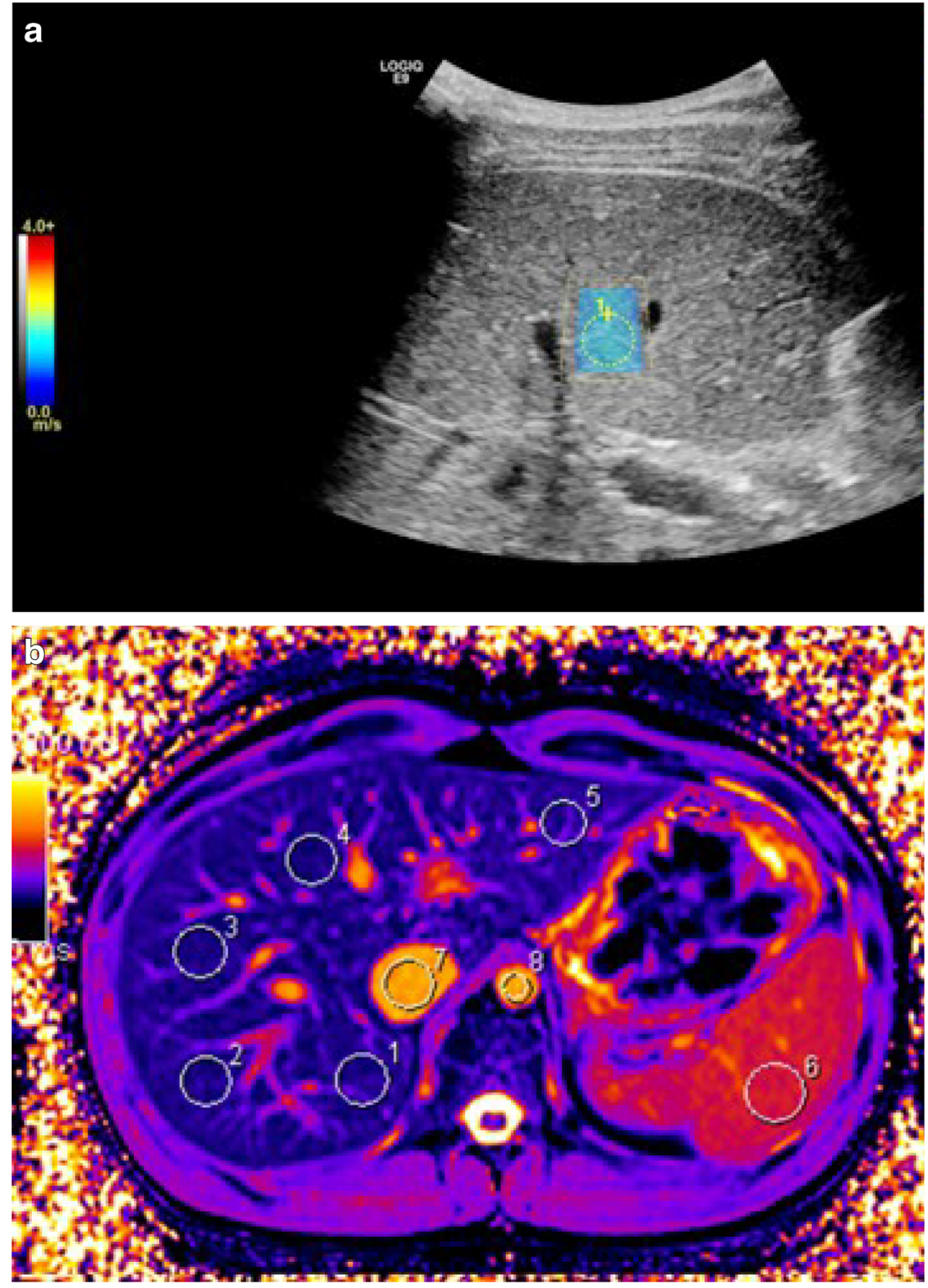

based on post-inversion recovery times and signal intensities. We measured the T1 of the blood pool in the inferior caval vein and abdominal aorta, avoiding hepatic tissue and vessel walls. For the liver, we used T1 values from the inferior caval vein and aorta (75\% and $25 \%$ ) to adjust for the double blood supply and low oxygenation of liver tissue [23]. For the spleen, we used only the T1 of the aorta as the blood pool value. We calculated the extracellular volume fraction based on pre-contrast, post-contrast and blood pool T1 values, and sampled hematocrit values within $24 \mathrm{~h}$ of the MR examination. We used a modification of the formula typically used for the myocardium, as follows [10]:

$E C V=(1$-hematocrit $) \frac{(1 / \text { postcontrastT1liver })-(1 / \text { nativeT1liver })}{(1 / \text { postcontrastT1blood })-(1 / \text { nativeT1blood })}$
Intraobserver variation was assessed in 15 randomly selected participants and controls. All measurements were performed blinded by one radiologist with 3 years of experience (K.J.T.). The analysis was repeated 4 weeks later. To evaluate interobserver variation, a second radiologist with 15 years of experience performed an assessment (C.dL.).

\section{Ultrasonography with shear-wave elastography}

Ultrasound 2-D shear-wave elastography was performed with a LogiqE9 (GE Healthcare, Chicago, IL) with a curved 1- to 6-MHz probe and fixed parameters. All subjects fasted for 3-4 h and rested for $10 \mathrm{~min}$ prior to elastography, and we recorded alcohol consumption in the previous week. This was to avoid falsely increased 
Fig. 2 Images of shear-wave elastography and native MR T1 mapping in a 15-year old girl with Fontan circulation. a Shear-wave elastography. The colors in the region of interest (yellow circle) indicate an increased value of $1.9 \mathrm{~m} / \mathrm{s}$. b Native MR T1 map shows an uneven color distribution. White circles indicate five regions of interest within the liver (1-5), one in the spleen (6), one in the inferior caval vein (7) and one in the aorta (8)
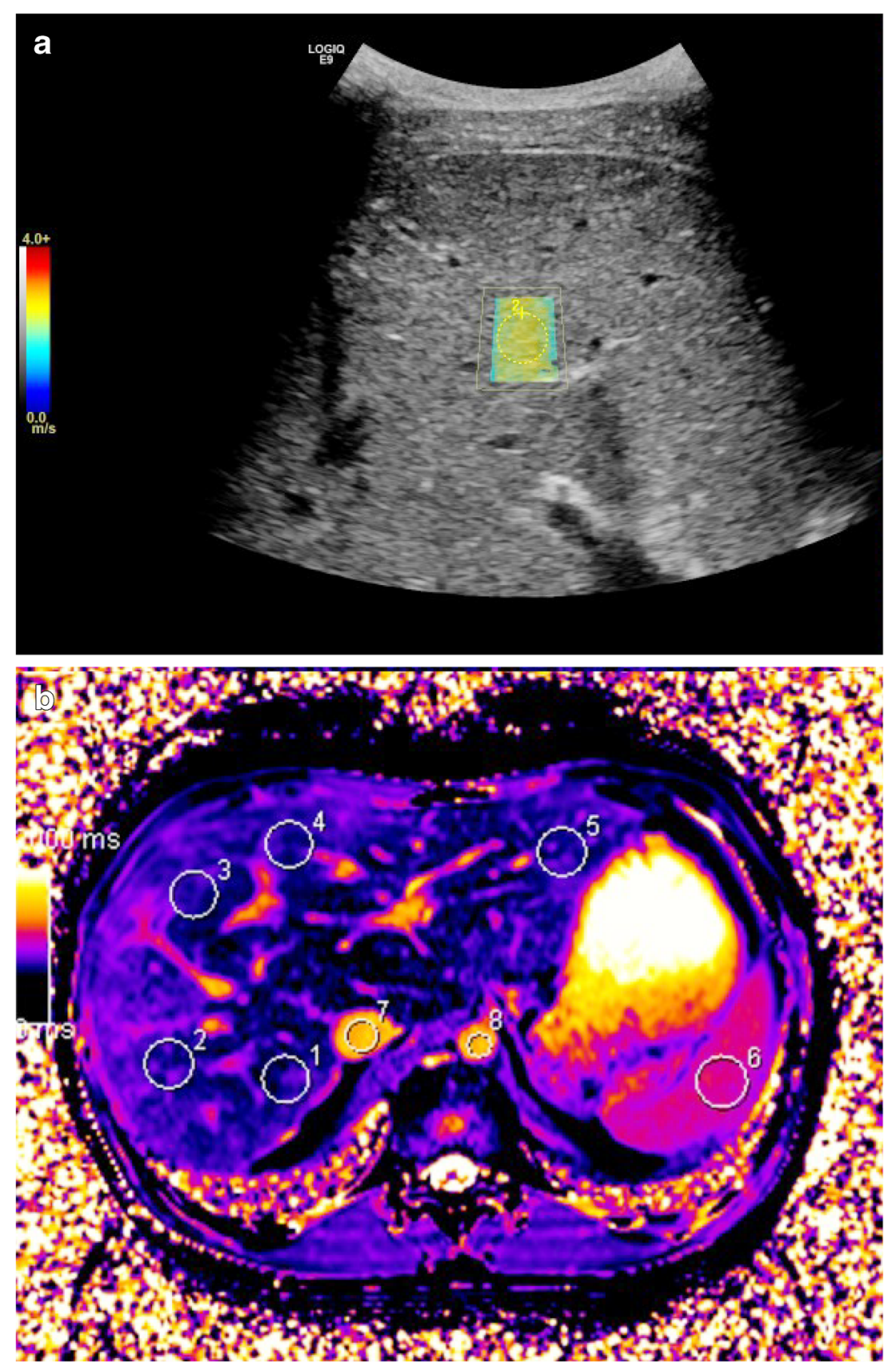

stiffness measurements from increased hepatic blood flow. Elastography was performed by two radiologists (K.J.T. and K.S.T., both with 5 years of experience). With the subject in a supine position, we achieved velocity sampling in the right liver lobe with an intercostal approach. Measurements were collected from a circular region of interest, $1 \mathrm{~cm}$ in diameter, at a depth of $3-4 \mathrm{~cm}$ from the liver surface. The median of five measurements $(\mathrm{m} / \mathrm{s})$ was recorded, with an interquartile range/median $<30 \%$ (Figs. 1 and 2). Interobserver variability was recorded in 10 control individuals.

\section{Hepatic serological biochemical markers}

Synthetic liver dysfunction was defined as an albumin concentration below the normal range in patients without proteinlosing enteropathy or an elevation in the international normalized ratio above 1.2 (in the absence of warfarin medication). Metabolic liver dysfunction was indicated by values outside the normal range for a given age for unconjugated bilirubin, bile acids, aspartate transaminase, alanine aminotransferase, alkaline phosphatase, gamma-glutamyl transferase, and lactate dehydrogenase. 


\section{Statistical analysis}

Continuous variables are presented as means \pm standard deviation (SD), for normally distributed data, or as medians and ranges for skewed data. Categorical variables are expressed as counts and percentages of the total. Groups were compared with the Student's $t$-test or Wilcoxon rank sum test, as appropriate for continuous variables, and with Fisher exact tests for categorical variables.

We assessed correlations between continuous and categorical parameters with univariate regression analyses. Twosided $P$-values $<0.05$ were considered statistically significant. Intra- and interobserver agreement were assessed with BlandAltman analyses [24]; results are expressed as the percentage bias and coefficient of variation. Statistical analyses were performed with SPSS version 25 (IBM, Armonk, NY).

\section{Results}

\section{Patient characteristics}

This study included 45 adolescent patients (median age: 16.5 years, range: $15.4-17.9$ years) and 15 healthy young adults (median age: 18.0 years, range: $18.0-21.6$ years). In 10 patients, MR could not be performed because of incompatible pacemaker devices or leads $(n=8)$ or claustrophobia $(n=2)$.

Normal reference values for hepatic T1 times and extracellular volume fractions were based on measurements in control subjects. Clinical characteristics of all participants are presented in Table 1. One patient had left isomerism with polysplenia, and one had situs inversus abdominis. These anomalies did not affect the quality or performance of MR T1 mapping or elastography. The craniocaudal spleen length was measured with MR; 11 patients had spleen lengths $>13 \mathrm{~cm}$ and 5 had frank splenomegaly $>14.5 \mathrm{~cm}$ (Table 2) [25].

Ascites was detected on MR in 15 patients. Protein-losing enteropathy had been diagnosed in three adolescents as part of a failing Fontan circulation, based on clinical symptoms (hypoalbuminemia, hypogammaglobulinemia, edema and ascites).

\section{Magnetic resonance $\mathrm{T} 2 *$, $\mathrm{T} 1$ mapping, and extracellular volume fractions in liver and spleen}

Nineteen adolescents had a dominant morphological single right ventricle, and 24 had a dominant morphological single left ventricle. Two had two functionally connected ventricles; these patients were excluded from the comparison of liver analyses between patients with different ventricular morphologies. Thirty-five participants underwent successful MR examinations without complications.
Native T1 times and hepatic extracellular volume fractions were significantly increased in the Fontan group compared to controls (Table 2; Fig. 3). In addition, T1 times were increased in adolescents with a single right ventricle compared to those with a single left ventricle. No other significant difference was found between the different ventricular morphologies. T1 times and spleen extracellular volume fractions were comparable between patients and controls (Table 2). No patient showed evidence of iron deposition in the liver; the mean T2* value was $32.0 \pm 3.6 \mathrm{~ms}$ [26]. Ascites was present in 15 patients, including the three with failing Fontan circulations.

\section{Ultrasound elastography in the liver}

Forty-five patients and 15 controls with no recent history of alcohol consumption underwent elastography. One patient could not hold the breath and was excluded because of unreliable measurements. Liver stiffness was elevated in all patients; the median velocity was $1.91 \pm 0.13 \mathrm{~m} / \mathrm{s}$ in patients and $1.20 \pm 0.10$ in controls $(P<0.001$; Table 2; Fig. 4).

\section{Observer variation in MR and elastography measurements}

The repeatability of liver MR measurements was tested with Bland-Altman plots in a subgroup of 15 patients and 15 controls. In the Fontan group, the $\mathrm{T} 1$ measurements showed moderate repeatability; the intraobserver bias was $0.03 \%$ (coefficient of variation: 1.5 ); the interobserver bias was $2.2 \%$ (coefficient of variation: 1.6 ). Controls had an intraobserver bias of $0.3 \%$ (and coefficient of variation: 0.6 ) and interobserver bias of $1.4 \%$ (coefficient of variation: 1.2) (Fig. 5). Variability in extracellular volume fractions are displayed in Online Supplementary Material 2. Interobserver variation analyses for US elastography were tested in 10 controls, with moderate reproducibility. The relative bias was $-3.4 \%$ (coefficient of variation: 7.0; Online Supplementary Material 2).

\section{Cardiac catheterization}

Among 45 patients, 43 underwent cardiac catheterization. Two patients had undergone cardiac catheterization within the last 2 years and showed no clinical indication; therefore, another catheterization was not performed. For one patient, catheterization was postponed for 3 months after MR or US elastography because of limited catheter lab availability. The mean central venous pressure was $13 \pm 3 \mathrm{mmHg}$, and the mean hepatic vein wedge pressure was $15 \pm 3 \mathrm{mmHg}$, with no difference between different ventricular morphologies (Table 1). 
Table 1 Patient characteristics

$\begin{array}{llllll}\text { Fontan } n=45 & \text { Control } n=15 & \begin{array}{l}P \text {-value } \\ \text { Fontan vs. control }^{\text {a }}\end{array} & \begin{array}{l}\text { Single left } \\ \text { ventricle } \\ \text { Fontan } n=24\end{array} & \begin{array}{l}\text { Single right } \\ \text { ventricle }\end{array} & \begin{array}{l}P \text {-value } \text { Fontan }^{\text {a }} \text { single } \\ \text { left vs. single right }\end{array} \\ & & \text { ventricle }\end{array}$

\begin{tabular}{|c|c|c|c|c|c|c|}
\hline \multicolumn{7}{|l|}{ Clinical } \\
\hline Age at MRI, in years ${ }^{b}$ & $16.7 \pm 0.6(15.4-17.9)$ & $19.2 \pm 1.2(18.0-21.6)$ & $<0.001$ & $16.5 \pm 0.6$ & $16.8 \pm 0.6$ & 0.1 \\
\hline Male gender ${ }^{c}$ & $28(61 \%)$ & $7(47 \%)$ & 0.4 & $14(58 \%)$ & $12(60 \%)$ & 0.5 \\
\hline Weight $(\mathrm{kg})^{\mathrm{b}}$ & $58.2 \pm 11.4$ & $64.7 \pm 14.8$ & 0.2 & $58.0 \pm 11.2$ & $62 \pm 11$ & 0.3 \\
\hline Body surface area $\left(\mathrm{m}^{2}\right)^{\mathrm{b}}$ & $1.6 \pm 0.2$ & $1.8 \pm 0.3$ & 0.08 & $1.6 \pm 0.2$ & $1.7 \pm 0.2$ & 0.2 \\
\hline Oxygen saturation $(\%)^{\mathrm{b}}$ & $95 \pm 2$ & - & - & $95 \pm 3$ & $95 \pm 2$ & 0.5 \\
\hline \multicolumn{7}{|l|}{ Surgical } \\
\hline Age at Fontan operation, in years ${ }^{\mathrm{b}}$ & $2.6 \pm 2.0$ & - & - & $2.8 \pm 2.1$ & $2.4 \pm 2.1$ & 0.5 \\
\hline $\begin{array}{l}\text { Interval MR/elastography } \\
\text { Fontan operation, in days }\end{array}$ & $5,021 \pm 1,076$ & & - & $5,036 \pm 757$ & $5,268 \pm 800$ & 0.3 \\
\hline \multicolumn{7}{|l|}{ Catheterization } \\
\hline Heart rate, in bpm $^{\mathrm{b}}$ & $74 \pm 15$ & & - & - & - & - \\
\hline $\begin{array}{l}\text { Peak systolic ventricular } \\
\text { pressure, in } \mathrm{mmHg}^{\mathrm{b}}\end{array}$ & $95 \pm 15$ & & & $98 \pm 18$ & $92 \pm 9$ & 0.2 \\
\hline $\begin{array}{l}\text { End-diastolic ventricular } \\
\text { pressure, in } \mathrm{mmHg}^{\mathrm{b}}\end{array}$ & $11 \pm 4$ & & - & $11 \pm 4$ & $11 \pm 4$ & 0.7 \\
\hline $\begin{array}{l}\text { Central venous pressure, } \\
\text { in } \mathrm{mmHg}^{\mathrm{b}}\end{array}$ & $13 \pm 3$ & & - & $13 \pm 3$ & $14 \pm 3$ & 0.1 \\
\hline $\begin{array}{l}\text { Hepatic vein wedge } \\
\text { pressure, in } \mathrm{mmHg}^{\mathrm{b}}\end{array}$ & $15 \pm 3$ & & - & $14 \pm 3$ & $16 \pm 3$ & 0.08 \\
\hline
\end{tabular}

${ }^{\text {a }} P$-value $<0.05$ is significant (bold)

${ }^{\mathrm{b}}$ Values are the mean \pm standard deviation (range)

${ }^{\mathrm{c}}$ Values are the number $(\%)$

\section{Hepatic serological markers}

Among the 45 adolescents with Fontan circulation, 40 showed only mild impairments, based on plasma hepatic markers (Table 3). Metabolic hepatic function was mildly abnormal in 40 patients (89\%), and synthetic function was mildly abnormal in $24(53 \%)$ patients. The international normalized ratio was increased (up to 1.3) in 5 patients. Of the 15 patients with ascites, 2 had reduced albumin levels ( $23 \mathrm{~g} / \mathrm{L}$; both had protein-losing enteropathy; Table 3).

Table 2 Ultrasound elastography of the liver and native T1 times and extracellular volume fractions of the liver and spleen

\begin{tabular}{|c|c|c|c|c|c|c|}
\hline & Fontan & Control $n=15$ & $\begin{array}{l}P \text {-value }{ }^{\text {a Fontan }} \\
\text { vs. control }\end{array}$ & $\begin{array}{l}\text { Single left } \\
\text { ventricle } n=24\end{array}$ & $\begin{array}{l}\text { Single right } \\
\text { ventricle } n=19\end{array}$ & $\begin{array}{l}P \text {-value }{ }^{\text {a }} \text { single left } \\
\text { vs. single right ventricle }\end{array}$ \\
\hline \multicolumn{7}{|l|}{ Shear-wave elastography } \\
\hline Liver median, in $\mathrm{m} / \mathrm{s}, n=45^{\mathrm{b}}$ & $1.91 \pm 0.14$ & $1.23 \pm 0.10$ & $<0.001$ & $1.93 \pm 0.14$ & $1.94 \pm 0.12$ & 0.7 \\
\hline \multicolumn{7}{|c|}{ MR, T2*, T1 mapping/extracellular volume fraction } \\
\hline Liver $\mathrm{T} 1$, in $\mathrm{ms}, n=35^{\mathrm{b}}$ & $774 \pm 44$ & $632 \pm 52$ & $<0.001$ & $756 \pm 49$ & $787 \pm 31$ & 0.03 \\
\hline $\begin{array}{l}\text { Liver extracellular volume } \\
\text { fraction, in } \%, n=35^{\mathrm{b}}\end{array}$ & $47.4 \pm 5.0$ & $34.6 \pm 3.8$ & $<0.001$ & $46.4 \pm 4.8$ & $48.0 \pm 5.4$ & 0.4 \\
\hline Spleen T1, in $\mathrm{ms}, n=35^{\mathrm{b}}$ & $1,169 \pm 62$ & $1,139 \pm 89$ & 0.2 & $1,159 \pm 52$ & $1,176 \pm 72$ & 0.4 \\
\hline $\begin{array}{l}\text { Spleen extracellular volume } \\
\text { fraction, in } \%, n=35^{\mathrm{b}}\end{array}$ & $30.7 \pm 3.1$ & $30.5 \pm 1.9$ & 0.7 & $30.8 \pm 3.1$ & $30.6 \pm 3.1$ & 0.8 \\
\hline Liver $\mathrm{T}^{*}$, in $\mathrm{ms}, n=32^{\mathrm{b}}$ & $32.0 \pm 3.6$ & $30.0 \pm 3.2$ & 0.05 & $32.1 \pm 4.0$ & $32.0 \pm 4.1$ & 0.8 \\
\hline Spleen $\mathrm{T} 2 *$, in $\mathrm{ms}, n=31^{\mathrm{b}}$ & $60 \pm 19$ & $49 \pm 10$ & 0.04 & $62 \pm 22$ & $60 \pm 15$ & 0.7 \\
\hline Spleen length, in $\mathrm{cm}^{\mathrm{b}}$ & $12 \pm 2(8-19)$ & & & & & \\
\hline
\end{tabular}

${ }^{\text {a }} P$-value $<0.05$ is significant (bold)

${ }^{\mathrm{b}}$ Values are the mean \pm standard deviation (range) 

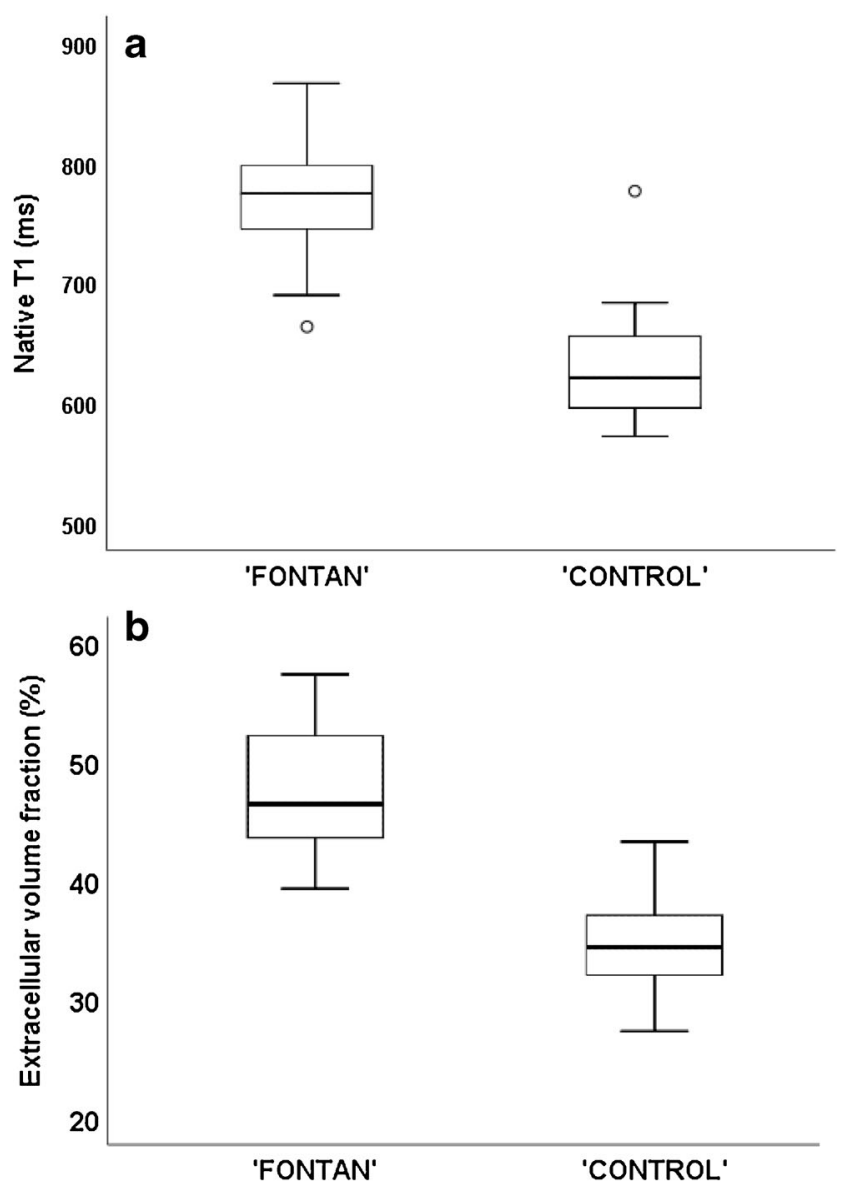

Fig. 3 Graphs show MR results in the Fontan group compared to the control group. a, b Native T1 times (a) and extracellular volume fractions (b) are higher in the Fontan group

\section{Associations between measured fibrosis indicators and clinical parameters}

In adolescents with Fontan circulation, the hepatic extracellular volume fraction was correlated with elastography $(\mathrm{r}=0.5$,

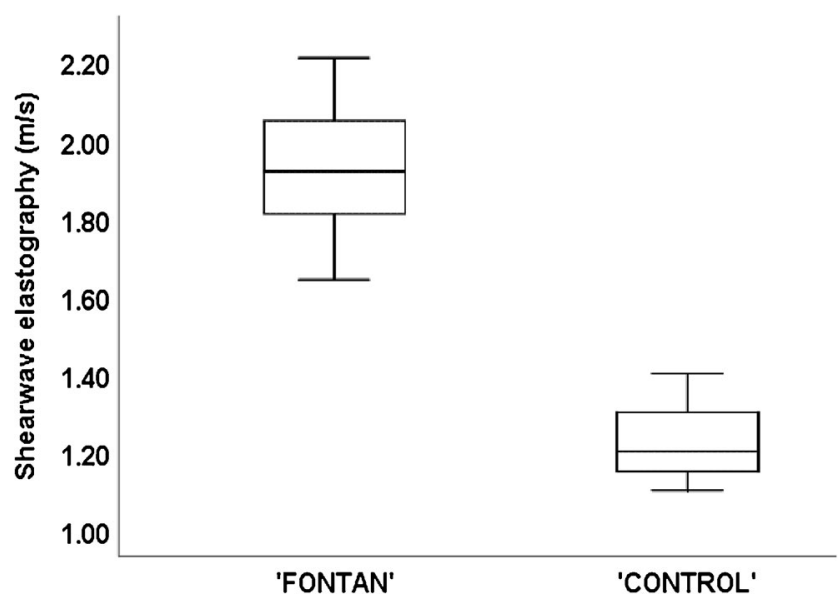

Fig. 4 Graph shows liver stiffness measured with US shear-wave elastography in the Fontan and control groups
$P=0.005)$, hepatic $\mathrm{T} 1$ times $(\mathrm{r}=0.5, P<0.001)$ and spleen extracellular volume fraction ( $\mathrm{r}=0.4, P=0.02$; Table 4$)$. In controls, the hepatic extracellular volume fraction was not correlated with elastography $(\mathrm{r}=0.3, P=0.2)$ or hepatic $\mathrm{T} 1$ times $(\mathrm{r}=0.2, P=0.4)$. The hepatic native $\mathrm{T} 1$ time was significantly correlated with central venous pressure $(\mathrm{r}=0.5, P=0.007)$ and liver vein wedge pressure $(\mathrm{r}=0.4, P=0.02)$. The hepatic extracellular volume fraction and elastography were not correlated with central venous pressure $(\mathrm{r}=0.3, P=0.1)$ or liver vein wedge pressure $(\mathrm{r}=0.3, P=0.2)$.

For patients, the native splenic $\mathrm{T} 1$ and extracellular volume fraction were positively correlated with central venous pressure ( $\mathrm{r}=0.4, P=0.03$, and $\mathrm{r}=0.5, P=0.02$, respectively) and liver vein wedge pressure ( $\mathrm{r}=0.5, P=0.006$, and $\mathrm{r}=0.5, P=0.03$, respectively; Table 4). The splenic extracellular volume fraction was also correlated with end-diastolic ventricular pressure $(\mathrm{r}=0.4, P=0.03)$.

Only one of the mildly abnormal hepatic markers was correlated with hepatic T1, extracellular volume fraction, or elastography: gammaglutamyl transferase was moderately correlated with hepatic extracellular volume fraction $(\mathrm{r}=0.4$, $P=0.04$, Table 4).

\section{Discussion}

The presence and severity of Fontan-associated liver disease play key roles in clinical decisions regarding reinterventions or the timing of a heart transplantation. Advanced liver disease might preclude transplantation, which is the only curative treatment for univentricular heart disease. Hence, reliable, accessible methods for detecting and monitoring the development of liver fibrosis are urgently needed.

This cross-sectional study of adolescents with Fontan circulation compared two new techniques for assessing hepatic fibrosis: MR T1 mapping and US shear-wave elastography. We found elevated MR and US elastography values in the liver, which indicate liver congestion or fibrosis, but normal values in the spleen. Moreover, correlations between elastography and MR relaxometry were inconsistent in our study groups. These methods were only correlated for extracellular volume fraction measurements in the Fontan group. The inconsistencies between US elastography and MR in detecting Fontan-associated hepatic changes indicate that these methods are not interchangeable in this patient group. Furthermore, increased central venous pressure correlated with hepatic native $\mathrm{T} 1$ times but not with elastography. Finally, we found no strong evidence that T1, extracellular volume fraction, or elastography correlates with serological hepatic markers.

The elevated hepatic T1, extracellular volume fraction, and elastography values confirmed previous findings from retrospective MR studies in adults and children with Fontan 

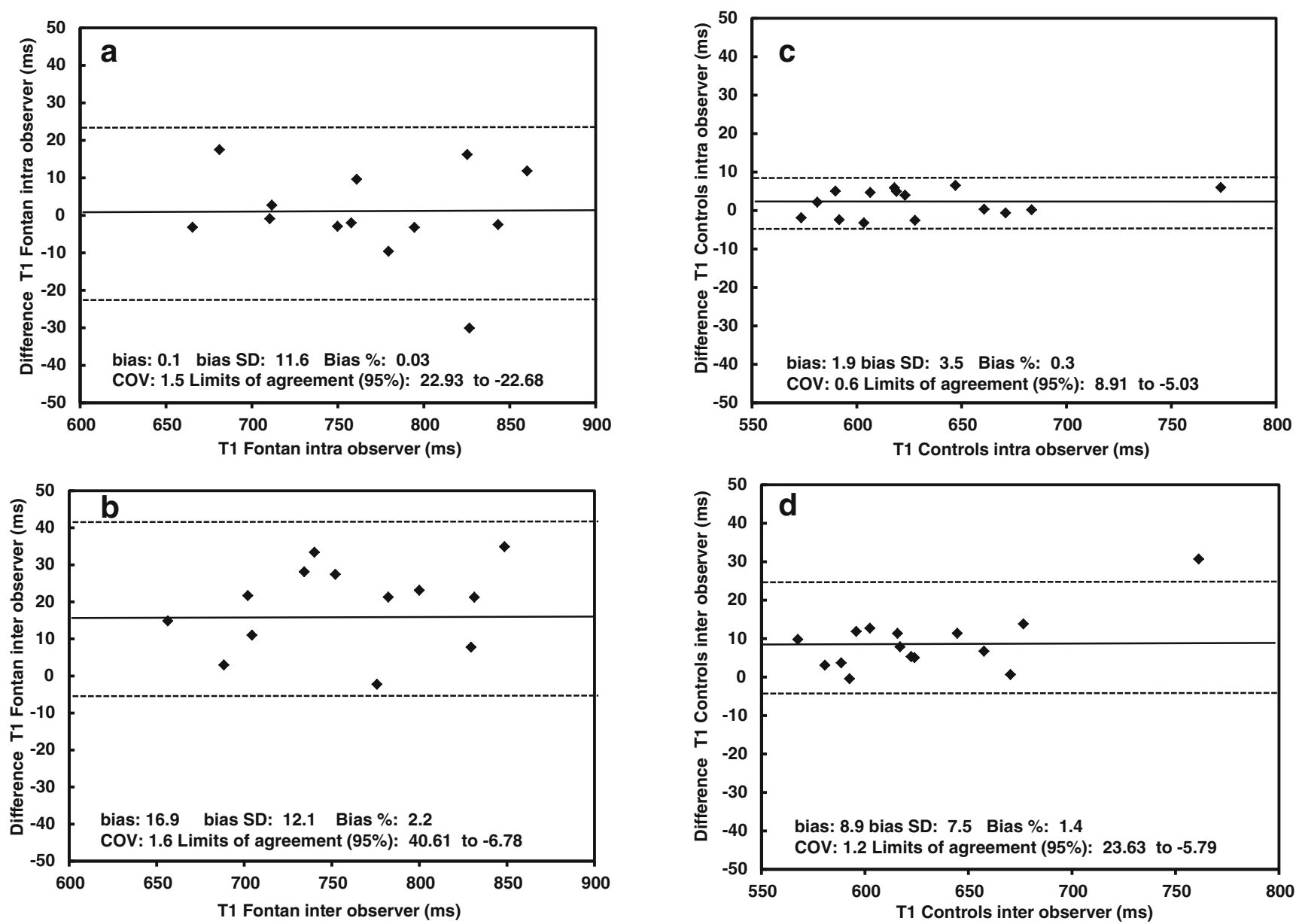

Fig. 5 Bland-Altman plots show observer variation results. a-d Intraobserver (a and $\mathbf{c}$ ) and interobserver (b and $\mathbf{d})$ agreements analyzed for MR native T1 times in the Fontan group $(\mathbf{a}, \mathbf{b})$ and the

control group (c, d). Results for the MR of extracellular volume fraction are shown in Online Supplementary Material 2. COV coefficient of variation

circulation [18, 21, 22] and studies on US and MR elastography after Fontan completion [19, 20, 27, 28]. The advantage of our study was the representative, unselected, relatively large national cohort of adolescents with Fontan

circulation. In addition, a dedicated axial liver MR sequence was used to cover more area in the heterogeneous liver tissue and to minimize MR-offset artifacts. In contrast, previous studies used cardiac MR sequences that covered small liver

Table 3 Hepatic biochemical markers in children with Fontan circulation

\begin{tabular}{lcc}
\hline $\boldsymbol{n = 4 5}$ & Mean \pm SD & Median \\
\hline Metabolic & & \\
Alanine aminotransferase & $31.7 \pm 14.0$ & 28 \\
Aspartate transaminase & $29.4 \pm 7.7$ & 28 \\
Gamma-glutamyl transferase & $72.2 \pm 37.7$ & 63 \\
Bilirubin & $13.7 \pm 9.2$ & 13 \\
Bile acid & $12.2 \pm 19.1$ & 6 \\
Lactate dehydrogenase & $181.8 \pm 30.2$ & 182 \\
Alkaline phosphatase & $134.9 \pm 51.0$ & 115 \\
Synthetic & & \\
Albumin & $45.8 \pm 5.1$ & 46 \\
International normalized ratio (without warfarin) & $1.2 \pm 0.1$ & 1.1 \\
\hline
\end{tabular}

$S D$ standard deviation 
Table 4 Correlations and univariate regression analysis results for patients with Fontan circulation

\begin{tabular}{|c|c|c|c|c|c|c|c|c|c|}
\hline & \multicolumn{3}{|c|}{ Liver native $\mathbf{T 1}$} & \multicolumn{3}{|c|}{ Liver extracellular volume fraction } & \multicolumn{3}{|c|}{ Liver elastography } \\
\hline & $\mathrm{r}$ & $P$-value & Confidence interval & $\mathrm{r}$ & $P$-value & Confidence interval & $\mathrm{r}$ & $P$-value & Confidence interval \\
\hline Height & & & & & & & 0.3 & 0.02 & $(0.01-0.01)$ \\
\hline Weight & & & & & & & 0.3 & 0.03 & $(0.01-0.01)$ \\
\hline Body surface area & & & & & & & 0.4 & 0.02 & $(0.04-0.50)$ \\
\hline T1 liver & & & & 0.6 & $<0.001$ & $(0.04-0.1)$ & & & \\
\hline T1 spleen & 0.4 & 0.02 & $(0.03-0.5)$ & 0.2 & 0.2 & & & & \\
\hline Extracellular volume fraction spleen & 0.2 & 0.2 & & 0.4 & 0.02 & $(0.14-1.2)$ & 0.1 & 0.5 & \\
\hline Liver elastography & 0.2 & 0.1 & & 0.5 & 0.005 & $(5.4-28.5)$ & & & \\
\hline Gammaglutamyl transferase & & & & 0.4 & 0.04 & $(0.001-1.2)$ & & & \\
\hline End-diastolic ventricular pressure & 0.2 & 0.4 & & 0.06 & 0.8 & & 0.03 & 0.9 & \\
\hline Peak systolic ventricular pressure & 0.2 & 0.4 & & 0.2 & 0.2 & & 0.1 & 0.5 & \\
\hline Liver vein wedge pressure & 0.4 & 0.02 & $(1.1-10.4)$ & 0.3 & 0.2 & & 0.3 & 0.1 & \\
\hline Central venous pressure & 0.5 & 0.007 & $(1.8-10.8)$ & 0.3 & 0.1 & & 0.2 & 0.3 & \\
\hline
\end{tabular}

regions. Moreover, in our cohort, elastography and MR were performed in conjunction under standardized conditions.

Jin et al. [29] compared these two techniques in adults with chronic liver disease. The authors found a good correlation between the extracellular volume fraction and elastography. This correlation strengthened with increasing histological stage severity [29]. Conversely, Ramachandran et al. [21] studied MR elastography in adults with Fontan circulation and reported that native T1 strongly correlated with MR elastography but not with extracellular volume fraction. Despite the homogeneous age of the patient group in our study, liver tissue changes were heterogeneous. However, native $\mathrm{T} 1$ times and extracellular volume fraction values in both patients and controls showed similar standard deviations. This heterogeneity in the parenchyma might represent focal variations in the vascular state of a normal liver. In the Fontan group with heterogeneous parenchyma, this was even more pronounced. Thus, it is important to measure several regions of interest to obtain a more representative evaluation of the liver. Indeed, elastography measures one region in the right liver lobe, and MR relaxometry covers different regions in both lobes. This difference in coverage might explain the modest correlation between the techniques.

We found a close relationship between central venous pressure and MR T1 mapping results. Elevated MR and elastography values might represent congestion, fibrosis, inflammation or a combination of these conditions. It is impossible with elastography to distinguish among these components with certainty. Specific MR sequences, like T2 mapping and T1 rho, might be helpful in discriminating fibrosis from edema in the future [30, 31]. On the other hand, a composite of hepatic congestion and fibrosis measures might be important, clinically, in the longitudinal follow-up of Fontan liver disease [32].
Multiple factors contribute to fibrosis development, but chronic venous congestion with elevated venous pressure plays a major role [33]. Other potential causes include low cardiac output, reduced oxygen delivery, inflammation and lymphatic stasis $[4,34]$. The exact stage of irreversible fibrosis/cirrhosis, and its importance in optimal heart transplantation timing, are under debate [5]. However, advanced-stage fibrosis/cirrhosis with possible hepatic malignancy might exclude cardiac transplantation or require a combined heart-liver transplant. Our young study population had normal splenic MR relaxometry values, which argued against a general inflammatory state and could be interpreted as the absence of splenic congestion/fibrosis. In turn, this finding also indicated normal portal venous pressure, which suggested a less-advanced liver fibrosis stage that might be reversible [4, 34, 35].

Previous studies, mainly in adults with Fontan circulation, showed that US and MR elastography were associated with increased central venous pressure $[19,27,36]$. In the present study, central venous pressure was correlated with hepatic and splenic T1 times but not extracellular volume fraction or elastography. MR T1 mapping could be used as an indicator of central venous pressure, which might minimize/prevent invasive ionizing catheterization. The lack of markedly elevated hepatic markers was consistent with previous findings in people with Fontan circulation, where normal levels of conventional biomarkers were observed despite severe structural liver changes [4, 5, 35].

This study had the following limitations. (1) Because of ethical considerations regarding informed consent, control individuals were slightly older than the patients, which might have influenced the normal values. (2) Only 35/45 patients could undergo MRI; thus, we could only compare the two techniques in 35 patients. (3) Elastography of the spleen was not accessible because artifacts made the measurements 
unreliable. (4) Liver biopsy was not performed because of the invasiveness and the high probability that samples would not be representative of the heterogeneous liver tissue in the adolescents with Fontan circulation. (5) The dual blood supply to the liver (75\% portal vein and $25 \%$ hepatic artery) provided predominantly venous oxygenation [23]. Because oxygen saturation is known to affect the T1 relaxation time [37], we attempted to avoid falsely elevated extracellular volume fraction calculations by measuring a split $\mathrm{T} 1$ blood pool (inferior caval vein and aorta) instead of measuring strictly arterial blood, as performed in the myocardium [10]. (6) T1 measurements in the Fontan versus control group revealed significant differences without overlap. However, when taking into account the moderate repeatability in the patient group, we acknowledge a possible risk of miscategorization in clinical routine. In addition, repeatability testing was restrained to two observers; thus, the variability in the T1 and extracellular volume fraction measurements might have been underestimated.

\section{Conclusion}

We compared hepatic MR relaxometry and US shear-wave elastography in adolescents with Fontan circulation. Both methods showed elevated markers suggestive of diffuse hepatic fibrosis or congestion. However, the detected changes differed between the methods; hence, they cannot be considered interchangeable. MRI appeared to be superior to US elastography because it provides a more global liver assessment, and, in addition, T1 mapping was closely related to central venous pressure. However, longitudinal, standardized studies with specific techniques for distinguishing congestion/ fibrosis might reveal objective MR evidence of the development of Fontan-associated liver disease.

Acknowledgments Open Access funding provided by University of Oslo (including Oslo University Hospital). We thank the MR technicians, Ailo Smuk and Åsmund Hagen Kersten, for valuable help in performing the MR examinations. This study was supported by grants from the Norwegian Association of Congenital Heart Disease (www.FFHB.no) and from the South-Eastern Norway Health Authority. The authors have control of all the data and information submitted for publication.

\section{Compliance with ethical standards}

\section{Conflicts of interest None}

Open Access This article is licensed under a Creative Commons Attribution 4.0 International License, which permits use, sharing, adaptation, distribution and reproduction in any medium or format, as long as you give appropriate credit to the original author(s) and the source, provide a link to the Creative Commons license, and indicate if changes were made. The images or other third party material in this article are included in the article's Creative Commons license, unless indicated otherwise in a credit line to the material. If material is not included in the article's Creative Commons license and your intended use is not permitted by statutory regulation or exceeds the permitted use, you will need to obtain permission directly from the copyright holder. To view a copy of this license, visit http://creativecommons.org/licenses/by/4.0/.

\section{References}

1. Fontan F, Baudet E (1971) Surgical repair of tricuspid atresia. Thorax 26:240-248

2. Mori M, Aguirre AJ, Elder RW et al (2014) Beyond a broken heart: circulatory dysfunction in the failing Fontan. Pediatr Cardiol 35: 569-579

3. Rychik J, Atz AM, Celermajer DS et al (2019) Evaluation and management of the child and adult with Fontan circulation: a scientific statement from the American Heart Association. Circulation. https://doi.org/10.1161/CIR.0000000000000696

4. Daniels CJ, Bradley EA, Landzberg MJ et al (2017) Fontanassociated liver disease: proceedings from the American College of Cardiology stakeholders meeting, October 1 to 2, 2015, Washington DC. J Am Coll Cardiol 70:3173-3194

5. Greenway SC, Crossland DS, Hudson M et al (2016) Fontanassociated liver disease: implications for heart transplantation. J Heart Lung Transplant 35:26-33

6. Serai SD, Trout AT, Miethke A et al (2018) Putting it all together: established and emerging MRI techniques for detecting and measuring liver fibrosis. Pediatr Radiol 48:1256-1272

7. Trout AT, Sheridan RM, Serai SD et al (2018) Diagnostic performance of MR elastography for liver fibrosis in children and young adults with a spectrum of liver diseases. Radiology 287:824-832

8. Wells ML, Fenstad ER, Poterucha JT et al (2016) Imaging findings of congestive hepatopathy. Radiographics 36:1024-1037

9. Westheim BH, Aagenaes I, Ostensen AB et al (2013) Effect of operator experience and frequency of procedure performance on complication rate after ultrasound-guided percutaneous liver biopsies. J Pediatr Gastroenterol Nutr 57:638-643

10. Messroghli DR, Moon JC, Ferreira VM et al (2017) Clinical recommendations for cardiovascular magnetic resonance mapping of $\mathrm{T} 1, \mathrm{~T} 2, \mathrm{~T} 2 *$ and extracellular volume: a consensus statement by the Society for Cardiovascular Magnetic Resonance (SCMR) endorsed by the European Association for Cardiovascular Imaging (EACVI). J Cardiovasc Magn Reson 19:75

11. Kozak MF, Redington A, Yoo SJ et al (2014) Diffuse myocardial fibrosis following tetralogy of Fallot repair: a T1 mapping cardiac magnetic resonance study. Pediatr Radiol 44:403-409

12. Kato A, Riesenkampff E, Yim D et al (2017) Pediatric Fontan patients are at risk for myocardial fibrotic remodeling and dysfunction. Int J Cardiol 240:172-177

13. Cassinotto C, Feldis M, Vergniol J et al (2015) MR relaxometry in chronic liver diseases: comparison of T1 mapping, T2 mapping, and diffusion-weighted imaging for assessing cirrhosis diagnosis and severity. Eur J Radiol 84:1459-1465

14. Yoon JH, Lee JM, Paek M et al (2016) Quantitative assessment of hepatic function: modified Look-Locker inversion recovery (MOLLI) sequence for T1 mapping on Gd-EOB-DTPA-enhanced liver MR imaging. Eur Radiol 6:1775-1782

15. Haimerl M, Utpatel K, Verloh N et al (2017) Gd-EOB-DTPAenhanced MR relaxometry for the detection and staging of liver fibrosis. Sci Rep 7:41429

16. Gao Y, Zheng J, Liang P et al (2018) Liver fibrosis with twodimensional US shear-wave elastography in participants with chronic hepatitis B: a prospective multicenter study. Radiology 289:407-415 
17. Kennedy P, Wagner M, Castera L et al (2018) Quantitative elastography methods in liver disease: current evidence and future directions. Radiology 286:738-763

18. Kazour I, Serai SD, Xanthakos SA, Fleck RJ (2018) Using T1 mapping in cardiovascular magnetic resonance to assess congestive hepatopathy. Abdom Radiol 43:2679-2685

19. DiPaola FW, Schumacher KR, Goldberg CS et al (2017) Effect of Fontan operation on liver stiffness in children with single ventricle physiology. Eur Radiol 27:2434-2442

20. Friedrich-Rust M, Koch C, Rentzsch A et al (2008) Noninvasive assessment of liver fibrosis in patients with Fontan circulation using transient elastography and biochemical fibrosis markers. J Thorac Cardiovasc Surg 135:560-567

21. Ramachandran P, Serai SD, Veldtman GR et al (2019) Assessment of liver T1 mapping in Fontan patients and its correlation with magnetic resonance elastography-derived liver stiffness. Abdom Radiol 44:2403-2408

22. de Lange C, Reichert MJE, Pagano JJ et al (2019) Increased extracellular volume in the liver of pediatric Fontan patients. J Cardiovasc Magn Reson 21:39

23. Yoo SJ, Prsa M, Schantz D et al (2014) MR assessment of abdominal circulation in Fontan physiology. Int J Cardiovasc Imaging 30: 1065-1072

24. Bland JM, Altman DG (1986) Statistical methods for assessing agreement between two methods of clinical measurement. Lancet $1: 307-310$

25. Prassopoulos P, Daskalogiannaki M, Raissaki M et al (1997) Determination of normal splenic volume on computed tomography in relation to age, gender and body habitus. Eur Radiol 7:246-248

26. Wood JC, Enriquez C, Ghugre N et al (2005) MRI R2 and R2* mapping accurately estimates hepatic iron concentration in transfusion-dependent thalassemia and sickle cell disease patients. Blood 106:1460-1465

27. Poterucha JT, Johnson JN, Qureshi MY et al (2015) Magnetic resonance elastography: a novel technique for the detection of hepatic fibrosis and hepatocellular carcinoma after the Fontan operation. Mayo Clin Proc 90:882-894
28. Serai SD, Wallihan DB, Venkatesh SK et al (2014) Magnetic resonance elastography of the liver in patients status-post Fontan procedure: feasibility and preliminary results. Congenit Heart Dis 9:714

29. Jin $\mathrm{K}$, Wang $\mathrm{H}$, Zeng $\mathrm{M}$ et al (2019) A comparative study of MR extracellular volume fraction measurement and two-dimensional shear-wave elastography in assessment of liver fibrosis with chronic hepatitis B. Abdom Radiol 44:1407-1414

30. Xie S, Li Q, Cheng Y et al (2017) Impact of liver fibrosis and fatty liver on T1rho measurements: a prospective study. Korean J Radiol 18:898-905

31. Luetkens JA, Klein S, Traber F et al (2018) Quantification of liver fibrosis at T1 and T2 mapping with extracellular volume fraction MRI: preclinical results. Radiology 288:748-754

32. Tellez L, Rodriguez de Santiago E, Minguez B et al (2020) Prevalence, features and predictive factors of liver nodules in Fontan surgery patients: the VALDIG Fonliver prospective cohort. J Hepatol 72:702-710

33. Schleiger A, Salzmann M, Kramer P et al (2020) Severity of Fontan-associated liver disease correlates with Fontan hemodynamics. Pediatr Cardiol 41:736-746

34. Tellez L, Rodriguez de Santiago E, Albillos A (2018) Fontanassociated liver disease. Rev Esp Cardiol 71:192-202

35. Munsterman ID, Duijnhouwer AL, Kendall TJ et al (2019) The clinical spectrum of Fontan-associated liver disease: results from a prospective multimodality screening cohort. Eur Heart J 40:10571068

36. Wallihan DB, Podberesky DJ, Marino BS et al (2014) Relationship of MR elastography determined liver stiffness with cardiac function after Fontan palliation. J Magn Reson Imaging 40:1328-1335

37. Portnoy S, Osmond M, Zhu MY et al (2017) Relaxation properties of human umbilical cord blood at 1.5 tesla. Magn Reson Med 77: $1678-1690$

Publisher's note Springer Nature remains neutral with regard to jurisdictional claims in published maps and institutional affiliations. 